\title{
Analysis of Millimeter-Wave Polarization Diverse MIMO Capacity
}

\author{
Nicholas P. Lawrence*, Brian W.-H. Ng*, Hedley J. Hansen*†, Derek Abbott* \\ *School of Electrical and Electronic Engineering, \\ University of Adelaide, SA 5005, Australia. \\ nicholas.lawrence@adelaide.edu.au \\ ${ }^{\dagger}$ RFT Group EWRD, DSTO, \\ PO Box 1500, Edinburgh, \\ SA 5111, Australia.
}

\begin{abstract}
Millimeter-waves offer the possibility of wide bandwidth and consequently high data rate for wireless communications. For both uni- and dualpolarized systems, signals sent over a link may suffer severe degradation due to antenna misalignment. Link robustness may be enhanced by the use of mutual orthogonality in three dimensions. In the general case of a Rician fading channel the inclusion of a third orthogonal dipole may provide a useful additional degree of freedom and allow high capacity communication in any unit vector direction.

Multiple-input multiple-output polarization diversity offers a way of improving signal reception without the limitations associated with spatial diversity. It additionally allows for polarization-time block coding at the transmitter and maximal ratio combining at the receiver.

We develop a novel Rician fading channel model for a millimeter-wave tri-orthogonal half-wave dipole transmitter and receiver employing polarization diversity. Our simulation extends analysis into a three-dimensional field of view representation fully exploiting individual channel paths. Capacity is observed to be more robust than that of a dual-polarized system over the majority of a field of view.
\end{abstract}

\section{INTRODUCTION}

Consumer wireless applications are driving demand for increased user capacity, reliability and throughput. Performance should ideally be consistent regardless of end user position and orientation. Multiple-input multiple-output (MIMO) signalling techniques exploiting spatial diversity through channel scattering have been widely adopted in wireless terrestrial applications to increase performance [1] [2] [3]. Currently employed systems utilise uni-polarized or dual-polarized propagation due to their ease of implementation. However, these types of polarization do not fully account for a three dimensional environment. From a simple geometrical analysis, performance is reliant on relative antenna positions. This becomes an important limiting factor as design frequency increases as constant linear transmit power becomes typically harder to maintain; the consequence of which is an exponential rise in system cost. Every possible design advantage needs to be sought.
Implementation at the widely adopted mobile communication frequencies in the low gigahertz region finds itself restricted to progressively complex processing techniques if it is to keep up with consumer demand. In the infra-red region propagation limitations are well documented. As a result, much interest has been given to the terahertz region where high data rate together with an unallocated portion of spectrum open the door to many possibilities [4]. Implementation at terahertz frequencies, in contrast to that at microwave frequencies, has been limited by available transmit power leading to line-of-sight (LOS) system design. In order to avoid link failure when LOS propagation is interrupted steerable dielectric mirrors have been introduced.

Innovative design at millimeter-wave frequencies offers many of the advantages of both microwave and terahertz frequencies while minimising the disadvantages [5] [6]. Firstly, the spectrum around this frequency has been allocated to wireless design over short distances. Secondly, any design and implementation may be influenced by well documented microwave techniques. Thirdly, available power at this frequency does not restrict the system to LOS propagation. Simple omnidirectional transmitters may be employed at the transmitter to increase coverage. Signal propagation may be further enhanced by the propagation environment itself.

Polarization diversity offers the benefit of MIMO signalling techniques in the instance of LOS propagation which is not always the case for a spatially diverse system. The benefit of a rich scattering environment of a fading channel may further enhance this performance [2].

The presented link is developed in three dimensions according to geometry in Figure 1. For a mutually tri-orthogonal antenna transmitter and receiver nine sub-channel paths are generated. Non-fading power transfer along each sub-channel path is given by the Friis formula, 


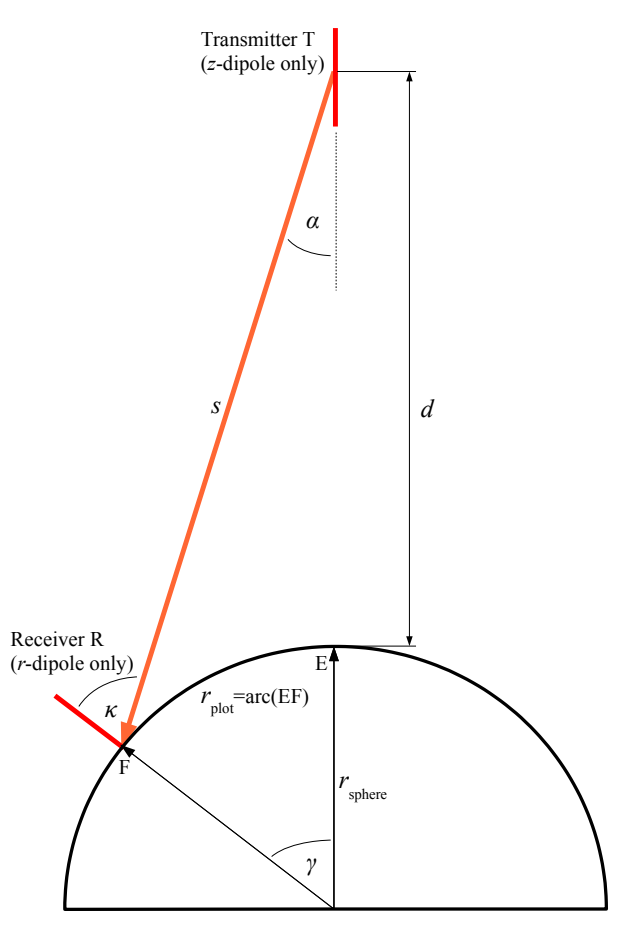

Fig. 1. Link geometry: three mutually orthogonal dipoles at both transmitter and receiver are introduced and are used to generate a transfer function. This allows for capacity data to be generated over a field of view, dependent on channel parameters. For clarity, only one dipole is shown at either end of the channel.

$$
\frac{P_{\mathrm{R}}}{P_{\mathrm{T}}}=G_{\mathrm{T}}\left(\phi_{\mathrm{T}}\right) G_{\mathrm{R}}\left(\phi_{\mathrm{R}}\right)\left(\frac{\lambda}{4 \pi s}\right)^{2} e_{\mathrm{pol}} L_{\text {atmos }}
$$

where $\mathrm{R}$ refers to the receiver dipole, $\mathrm{T}$ refers to the transmitter dipole, $P$ is power, $G$ is dipole gain, $\lambda$ is transmitted wavelength, $d$ is orbit height, and $s$ is separation of transmitter and receiver. Polarization mismatch, $e_{\mathrm{pol}}$, is between a dipole at the transmitter and at the receiver. Atmospheric attenuation, $L_{\text {atmos }}$, is due to the interaction of the electromagnetic signal with oxygen molecules at $60 \mathrm{GHz}$ and is given here as $15 \mathrm{dBkm}^{-1}$.

\section{Results}

Simulation is performed over the entire field of view (FoV) for the case of mutually orthogonal dipoles in three dimensions at transmitter and receiver; the former being fixed while the latter moves on a spherical surface to demonstrate both angular and spatial divergence. The extreme case of severe fading channel capacity is illustrated.

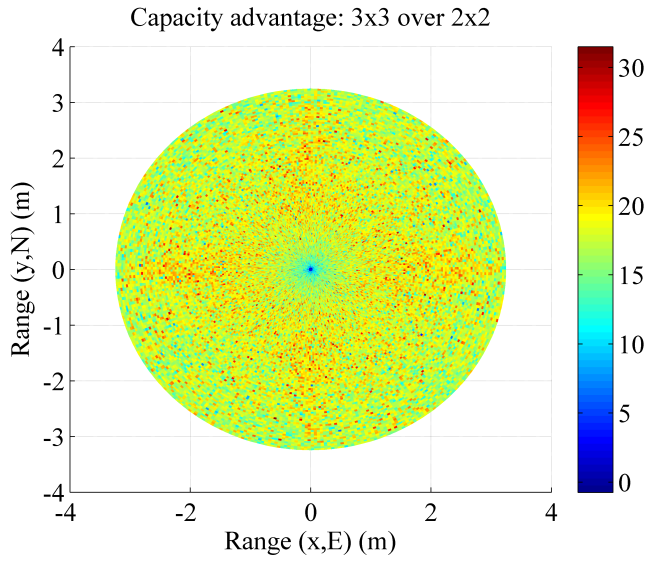

Fig. 2. Rayleigh channel capacity advantage (bits/s/Hz): triorthogonality over dual-orthogonality (frequency $=60 \mathrm{GHz}$, bandwidth $=7 \mathrm{GHz}$, transmit power $=40 \mathrm{dBm}$, propagation length $=$ $1 \mathrm{~m}$, sphere radius $=6 \mathrm{~m}$ ).

\section{Summary}

Excellent agreement is found at the FoV centre with both simulated and measured capacity analysis given in line graph format in [7] and [8]. Our simulation extends analysis into a three-dimensional FoV representation fully exploiting 9 individual channel paths. Our model allows the user to view capacity and hence system performance in a FoV format; useful in the instance of misaligned antennas and user movement. Tri-orthogonal capacity is observed as being more robust over the FoV, reducing antenna misalignment issues. The model may be readily expanded into low earth orbit (LEO) satellite channel modelling as well as state-of-the-art applications at terahertz frequencies.

\section{REFERENCES}

[1] W. Jakes, Microwave Mobile Communications. New York, NY: Wiley, 1974.

[2] A. Goldsmith, Wireless Communications. Cambridge, UK: Cambridge University Press, 2005.

[3] A. Paulraj, D. Gore, R. Nabar, and H. Bolcskei, "An overview of MIMO communications-a key to gigabit wireless," Proceedings of the IEEE, vol. 92, no. 2, pp. 198-218, 2004.

[4] T. Kleine-Ostmann and T. Nagatsuma, "A review on terahertz communications research," Infrared Millmetre Terahertz Waves, vol. 32, pp. 143-171, 2011.

[5] T. Rappaport, J. Murdock, and F. Gutierrez, "State of the art in $60-\mathrm{GHz}$ integrated circuits and systems for wireless communications," Proceedings of the IEEE, vol. 99, no. 8, pp. 1390-1436, 2011, DOI: 10.1109/JPROC.2011.2143650.

[6] T. Rappaport, "The coming renaissance of the wireless communications age," 2014, available at: http://nyuwireless.com/ NYSWAX.pdf.

[7] J. Wang, H. Zhang, L. Tingting, and T. Gulliver, "Capacity of $60 \mathrm{GHz}$ wireless communication systems over fading channels," Journal of Networks, vol. 7, no.1, pp. 203-209, 2012.

[8] C.-Y. Chiu, J.-B. Yan, and R. Murch, "Compact three-port orthogonally polarized MIMO antennas," IEEE Antennas and Wireless Propagation Letters, vol. 6, pp. 619-622, 2007. 